\title{
Using Relative Entropy to Find Optimal Approximations: an Application to Simple Fluids*
}

\author{
Chih-Yuan Tseng ${ }^{\dagger}$ \\ Graduate Institute of Systems Biology and Bioinformatics, \\ National Central University, Jhongli 320, Taiwan \\ Ariel Caticha $\ddagger$ \\ Department of Physics, University at Albany-SUNY \\ Albany, NY 12222 USA
}

\begin{abstract}
We develop a maximum relative entropy formalism to generate optimal approximations to probability distributions. The central results consist in (a) justifying the use of relative entropy as the uniquely natural criterion to select a preferred approximation from within a family of trial parameterized distributions, and (b) to obtain the optimal approximation by marginalizing over parameters using the method of maximum entropy and information geometry. As an illustration we apply our method to simple fluids. The "exact" canonical distribution is approximated by that of a fluid of hard spheres. The proposed method first determines the preferred value of the hard-sphere diameter, and then obtains an optimal hard-sphere approximation by a suitably weighed average over different hard-sphere diameters. This leads to a considerable improvement in accounting for the soft-core nature of the interatomic potential. As a numerical demonstration, the radial distribution function and the equation of state for a Lennard-Jones fluid (argon) are compared with results from molecular dynamics simulations.
\end{abstract}

Keyword: Approximation method, Maximum entropy, Marginalization, Simple fluids, Hard sphere approximation PACS: 05.20.Gg, 05.20.Jj

\section{Introduction}

A common problem in statistical physics is that the probability distribution functions (PDFs) are always too complicated for practical calculations and we need to replace them by more tractable approximations. A possible solution is to identify a family of trial distributions $\{p(x)\}$, where $\mathrm{x}$ are parameters characterized systems and select the member of the family that is closest to the exact distribution $P(x)$. The problem, of course, is that it is not clear what one means by 'closest'. One could minimize

$$
\int d x[p(x)-P(x)]^{2}
$$

\footnotetext{
* Accepted for publication in Physica A, 2008

${ }^{\dagger} \mathrm{He}$ is cuurently in the transition to Department of Oncology, University of Alberta, Edmonton AB T6G 1Z2; E-mail: richard617@gmail.com

‡E-mail: ariel@albany.edu
} 
but why this particular functional and not another? And also, why limit oneself to an approximation by a single member of the trial family? Why not consider a linear combination of the trial distributions, some kind of average over the trial family? But then, how should we choose the optimal weight assigned to each $p(x)$ ? We propose to tackle these questions using the method of Maximum relative Entropy (which we abbreviate as ME) and information geometry 1]. The ME method, which is developed in [2]- 8], has historical roots in the earlier method of maximum entropy that was pioneered by E. T. Jaynes and is commonly known as MaxEnt [9]. The ME method is designed for updating probabilities from arbitrary priors for information in the form of arbitrary constraints and it includes Bayes' rule and the older MaxEnt as special cases [7, 8].

The purpose of this paper is to develop a ME based method to generate optimal approximations (brief accounts of some of the results discussed below have previously been presented in [10] and [11]). The general formalism, which is the main result of this paper, is developed in section 2 In section 2.1 we justify the use of relative entropy as the unique and natural criterion to select the preferred approximation, which is labeled by some parameters. The optimal approximation is obtained in section 2.2 by marginalizing over the variational parameters. A suitably weighed average over the whole family of trial distributions with the optimal weight provides an optimal approximation.

In the second part of the paper we demonstrate the proposed formalism by applying it to simple classical fluids, a well studied field in the past [12]-15]. To approximate the behavior of simple fluids we chose trial distributions that describe hard spheres [12]-[15] (section [3). The ME formalism is first used (section 4.1) to select the preferred value of the hard-sphere diameter. This is equivalent to applying the Bogoliubov variational principle and reproduces the results obtained by Mansoori et al. [16] whose variational principle was justified by a very different argument.

An advantage of the variational or the ME methods over the perturbative approaches such as Barker and Henderson (BH) [12] and of Weeks, Chandler and Anderson (WCA) [13] is that there is no need for ad hoc criteria dictating how to separate the intermolecular potential into a strong short range repulsion and a weak long range attraction. On the other hand, a disadvantage of the standard variational approach is that it fails to take the softness of the repulsive core into account. At high temperatures this leads to results that are inferior to the perturbative approaches.

In the standard variational a single preferred value of the hard-sphere diameter is selected. But, as discussed in [7] and [17, in the ME method non-preferred values are not completely ruled out. This allows us (in section 4.2) to marginalize over hard sphere diameter to obtain an optimal hardsphere approximation with a suitable weighting. That leads to significant improvements over the standard variational method.

In section 5 we test our method by comparing its predictions for a Lennard-Jones model for argon with molecular dynamics simulation data ([18, 19]). We find that the ME predictions for thermodynamic variables and for the radial distribution function are considerable improvements over the standard Bogoliubov variational result, and are comparable to the perturbative results [12] [13]. (For a recent discussion of some of the strengths and limitations of the perturbative approach see [20.).). Despite the shortcomings of perturbation theory it remains very popular because it provides quantitative insights at a much lower computational cost than dynamical simulations. For recent applications to the glass transition and other more complex systems see 21] - 25]. Although in this work the ME is not applied to such complex problems one may fully expect that the information theory based ME method will yield insights not only the thermodynamic behavior of complex systems but also about the approximation methods needed to analyze them. Finally, our conclusions and some remarks on further improvements are presented in section 6 .

\section{General formalism of ME optimal approximations}

Consider a system with microstates labeled by $q$ (for example, the location in phase space or perhaps the values of spin variables). Let the probability that the microstate lies within a particular range 
$d q$ be given by the intractable canonical distribution

$$
P(q) d q=\frac{e^{-\beta H(q)}}{Z} d q,
$$

where $Z=\int d q e^{-\beta H(q)} \stackrel{\text { def }}{=} e^{-\beta F}$ is partition function, in which $H(q)$ is the Hamiltonian and free energy $F$ is defined through this partition function. The goal is to generate an approximation $p(q)$ that is optimal in the sense that is "closest" to the "exact" distribution $P(q)$. It includes two steps. The first is to select a preferred trial distribution from a family of trials $p^{\prime}(q \mid \theta)$, each member of the trial family being labeled by one or more parameters $\theta$. More generally, one could define the trial family in a non-parametric way by specifying various constraints. The second is to marginalize over parameters $\theta$ to obtain an optimal distribution.

\subsection{Entropic criterion for selecting preferred tractable PDFs}

Relative entropy as the selection criterion. The selection of the preferred approximation is achieved by ranking the distributions $p(q)$ according to increasing preference, a real number $\mathcal{D}[p]$ which we call the "entropy" of $p$. The numbers $\mathcal{D}[p]$ are such that if $p_{1}$ is preferred over $p_{2}$, then $\mathcal{D}\left[p_{1}\right]>\mathcal{D}\left[p_{2}\right]$. Thus, by design, the "preferred" approximation $p$ is that which maximizes the entropy $\mathcal{D}[p]$.

Next we determine the functional form of $\mathcal{D}[p]$. This is the general rule that provides the criterion for preference; in our case it defines what we mean by the "closest" or "preferred" approximation. The basic strategy [3] is one of induction: (1) if a general rule exists, then it must apply to special cases; (2) if in a certain special case we know which is the best approximation, then this knowledge can be used to constrain the form of $\mathcal{D}[p]$; and finally, (3) if enough special cases are known, then $\mathcal{D}[p]$ will be completely determined.

The known special cases are called the "axioms" of ME and they reflect the conviction that whatever information was originally codified into the exact $P(q)$ is important and should be preserved. The selected trial distribution should coincide with the exact one as closely as possible and one should only tolerate those minimal changes that are demanded by the information that defines the family of trials. Three axioms and their consequences are listed below. Detailed proofs and more extensive comments are given in [7] and [8.

Axiom 1: Locality. Local information has local effects. If the constraints that define the trial family do not refer to a certain domain $D$ of the variable $q$, then the conditional probabilities $p(q \mid D)$ need not be revised, $p(q \mid D)=P(q \mid D)$. The consequence of the axiom is that non-overlapping domains of $q$ contribute additively to the entropy: $\mathcal{D}[p]=\int d q F(p(q))$ where $F$ is some unknown function.

Axiom 2: Coordinate invariance. The ranking should not depend on the system of coordinates. The coordinates that label the points $q$ are arbitrary; they carry no information. The consequence of this axiom is that $\mathcal{D}[p]=\int d q p(q) f(p(q) / m(q))$ involves coordinate invariants such as $d q p(q)$ and $p(q) / m(q)$, where the function $m(q)$ is a density, and both functions $m$ and $f$ are, at this point, unknown.

Next we make a second use of Axiom 1 (locality). When there are no constraints at all and the family of trials includes the exact $P(q)$ the selected trial should coincide with $P(q)$; that is, the best approximation to $P(q)$ is $P(q)$ itself. The consequence is that up to normalization the previously unknown density $m(q)$ is the exact distribution $P(q)$.

Axiom 3: Consistency for independent subsystems. When a system is composed of subsystems that are independent it should not matter whether the approximation procedure treats them separately or jointly. Specifically, if $q=\left(q_{1}, q_{2}\right)$, and the exact distributions for the subsystems, $P_{1}\left(q_{1}\right)$ and $P_{2}\left(q_{2}\right)$, are respectively approximated by $p_{1}\left(q_{1}\right)$ and $p_{2}\left(q_{2}\right)$, then the exact distribution for the whole system $P_{1}\left(q_{1}\right) P_{2}\left(q_{2}\right)$ should be approximated by $p_{1}\left(q_{1}\right) p_{2}\left(q_{2}\right)$. This axiom restricts the function $f$ to be a logarithm. 
The overall consequence of these axioms is that the trial approximations $p(q)$ should be ranked relative to the exact $P(q)$ according to their (relative) entropy,

$$
\mathcal{D}[p \mid P]=-\int d q p(q) \log \frac{p(q)}{P(q)} .
$$

The derivation has singled out the relative entropy $\mathcal{D}[p \mid P]$ as the unique functional to be used for the purpose of selecting a preferred approximation. Other functionals, may be useful for other purposes, but they are not a generalization from the simple cases described in the axioms above.

Remark. Suppose a member of a family of trial canonical distributions $p^{\prime}(q \mid \theta)$ with Hamiltonian $H(q \mid \theta)$ that are conditional probability distributions and depend on parameters $\theta=\left\{\theta^{1}, \ldots, \theta^{n}\right\}$, are given by

$$
p^{\prime}(q \mid \theta) d q=\frac{e^{-\beta H(q \mid \theta)}}{Z_{\theta}} d q
$$

where $Z_{\theta}=\int d q e^{-\beta H(q \mid \theta)} \stackrel{\text { def }}{=} e^{-\beta F_{\theta}}$, in which free energy $F_{\theta}$ is also defined. The preferred trial is then selected by maximizing $\mathcal{D}\left[p^{\prime} \mid P\right]$. Substituting Eq. (2) and Eq. (4) into Eq. (3) gives,

$$
\mathcal{D}\left[p^{\prime} \mid P\right]=\beta\left(\left\langle H_{\theta}-H\right\rangle_{\theta}-F_{\theta}+F\right),
$$

where $\langle\ldots\rangle_{\theta}$ refers to averages computed with the distribution $p(q \mid \theta)$. The inequality $\mathcal{D}\left[p^{\prime} \mid P\right] \leq 0$, can then be written as

$$
F \leq F_{\theta}+\left\langle H-H_{\theta}\right\rangle_{\theta} .
$$

Thus, maximizing $\mathcal{D}\left[p^{\prime} \mid P\right]$ is equivalent to minimizing the quantity $F_{\theta}+\left\langle H-H_{\theta}\right\rangle_{\theta}$. This form of the variational principle and its use to generate approximations is well known. It is usually associated with the name of Bogoliubov [26] and it is the main technique to generate mean field approximations for discrete systems of spins on a lattice.

\subsection{Marginalization for optimal PDF}

The extent to which the preferred $\theta$ is preferred over other values ([7], [17]) is expressed by the probability of $\theta, p(\theta)$. The original ME problem of assigning a probability to $q$ is now broadened into assigning probabilities to both $q$ and $\theta$. In this section we use ME again to find the preferred joint distribution $p_{J}(q, \theta)=p(\theta) p(q \mid \theta)$. Note that this is the kind of problem where the Bayesian interpretation of probabilities is essential. Within a frequentist interpretation it makes no sense to talk about $p(\theta)$ or about $p(q \mid \theta)$ because $\theta$ is not a random variable; the value of $\theta$ is unknown but it is not random.

To proceed we must ask a question. What is the prior distribution, that is, what do we know about $q$ and $\theta$ before the trial family is specified?

The joint prior $m(q, \theta)$ can be expressed according to the product rule as, $m(q, \theta)=m(q \mid \theta) m(\theta)$, where $m(q \mid \theta)$ is conditional probability of observing system in state $q$ given parameter $\theta$. Our goal is to determine the preferred $p_{J}(q, \theta)$ that is closest to the prior $m(q, \theta)$ that reflects our initial knowledge about $q$ and ignorance about the $\theta$ s. Initially we know nothing about $\theta$, not even how it is related to $q$. The prior that represents this state of knowledge is a product

$$
m(q, \theta)=P(q) \mu(\theta) .
$$

Indeed, when $m(q, \theta)$ is a product no correlations between $\theta$ and $q$ are introduced which means that information about $q$ tells us nothing about $\theta$ and vice versa. The first factor in $m(q, \theta)$ reflects our prior knowledge about $q$ : the distribution for $q$ is known to be the exact $P(q)$. The second factor reflects our complete ignorance about $\theta$ : we choose $\mu(\theta)$ to be as uniform as possible. Our method applies whether $\theta$ is a discrete or a continuous variable. When $\theta$ is a continuous variable. 
Then the uniform distribution $\mu(\theta)$ is such that makes equal volumes in $\theta$ space equally likely. To define these volumes we apply method of information geometry [1] and note that distances in $\theta$ space are uniquely defined because the $\theta$ s are labels on probability distributions. Cases where $\theta$ is a discrete variable are simpler. The relevant entropies involve sums over $\theta_{i}$ rather than integrals and the natural uniform distribution is $\mu\left(\theta_{i}\right)=$ constant. In what follows we concentrate on the more challenging continuous case. The unique distance between $\theta$ and $\theta+d \theta$ is given by the Fisher-Rao metric [1], $d \ell^{2}=\gamma_{i j} d \theta^{i} d \theta^{j}$, where

$$
\gamma_{i j}=\int d q p(q \mid \theta) \frac{\partial \log p(q \mid \theta)}{\partial \theta^{i}} \frac{\partial \log p(q \mid \theta)}{\partial \theta^{j}} .
$$

Accordingly, the volume of a small region $d \theta$ is $\gamma^{1 / 2}(\theta) d \theta$, where $\gamma(\theta)$ is the determinant of $\gamma_{i j}$. Up to an irrelevant normalization, the distribution $\mu(\theta)$ that is uniform in $\theta$ is given by $\mu(\theta)=\gamma^{1 / 2}(\theta)$.

The preferred approximation $p_{J}(q, \theta)$ to the joint distribution $P(q) \gamma^{1 / 2}(\theta)$ is then obtained maximizing the entropy

$$
\mathcal{D}\left[p_{J} \mid \gamma^{1 / 2} P\right]=-\int d q d \theta p(\theta) p_{\theta}(q) \log \frac{p(\theta) p_{\theta}(q)}{\gamma^{1 / 2}(\theta) P(q)},
$$

by varying $p(\theta)$ subject to $\int d \theta p(\theta)=1$. The final result for the probability that $\theta$ lies within the small volume $\gamma^{1 / 2}(\theta) d \theta$ is

$$
p(\theta) d \theta=\frac{1}{\zeta} e^{\mathcal{D}\left[p_{\theta} \mid P\right]} \gamma^{1 / 2}(\theta) d \theta,
$$

where $\mathcal{D}\left[p_{\theta} \mid P\right]$ is given in Eq. (5) and $\zeta$ is a normalization constant. Note also that the density $\exp \mathcal{D}\left[p_{\theta} \mid P\right]$ is a scalar function and the presence of the Jacobian factor $\gamma^{1 / 2}(\theta)$ makes Eq. (10) manifestly invariant under changes of the coordinates $\theta$. Eq. (10) expresses the degree to which values of $\theta$ away from the preferred value are ruled out; it tells us that the preferred value of $\theta$ is that which maximizes the probability density $\exp \mathcal{D}\left[p_{\theta} \mid P\right]$.

Finally, now that we have determined the preferred joint distribution $p_{J}(q, \theta)=p(\theta) p(q \mid \theta)$ we can marginalize $\theta$ and use the average

$$
\bar{p}(q)=\int d \theta p(\theta) p(q \mid \theta)
$$

as the best approximation we can construct out of the given trial family. This approximation is expected to be better than any individual $p(q \mid \theta)$ for the same reason that the mean is expected to be a better estimator than the mode - it minimizes the variance.

This concludes the first part of our paper. To summarize: our main results consist in the justification of the relative entropy Eq. (3) as the uniquely natural functional to select the preferred approximations and the derivation of a quantitative measure of the degree to which the various trials are preferred, Eq. (10). The final result for the best approximation is Eq. (11).

Next we illustrate how this ME formalism is used in a specific example: simple fluids.

\section{ME optimal hard-sphere approximation for simple fluids}

\subsection{Basic features of simple fluids}

A simple fluid composed of $N$ single atom molecules is described by the Hamiltonian

$$
H\left(q_{N}\right)=\sum_{i=1}^{N} \frac{p_{i}^{2}}{2 m}+U \quad \text { with } \quad U=\sum_{i>j}^{N} u\left(r_{i j}\right),
$$


where $q_{N}=\left\{p_{i}, r_{i} ; i=1, \ldots, N\right\}$ and the many-body interactions are approximated by a pair interaction, $u\left(r_{i j}\right)$ where $r_{i j}=\left|r_{i}-r_{j}\right|$. The probability that the positions and momenta of the molecules lie within the phase space volume

$$
d q_{N}=\frac{1}{N ! h^{3 N}} \prod_{i=1}^{N} d^{3} p_{i} d^{3} r_{i}
$$

is given by canonical distribution

$$
P_{f}\left(q_{N}\right) d q_{N}=\frac{1}{Z_{f}} e^{-\beta H\left(q_{N}\right)} d q_{N},
$$

where $Z_{f}=\int d q_{N} e^{-\beta H\left(q_{N}\right)}$. For fluids dominated by pair interactions most thermodynamic quantities of interest can be written in terms of the one- and two-particle density distributions

$$
n(r)=\langle\hat{n}(r)\rangle \quad \text { and } \quad n^{(2)}\left(r_{1}, r_{2}\right)=\left\langle\hat{n}^{(2)}\left(r_{1}, r_{2}\right)\right\rangle
$$

where

$$
\hat{n}(r)=\sum_{i} \delta\left(r-r_{i}\right)
$$

and

$$
\hat{n}^{(2)}\left(r_{1}, r_{2}\right)=\sum_{i, j(i \neq j)} \delta\left(r_{1}-r_{i}\right) \delta\left(r_{2}-r_{j}\right) .
$$

The two-particle correlation function,

$$
g\left(r_{1}, r_{2}\right)=\frac{n^{(2)}\left(r_{1}, r_{2}\right)}{n\left(r_{1}\right) n\left(r_{2}\right)}
$$

measures the extent to which the structure of liquids deviates from complete randomness. If the fluid is homogeneous and isotropic $n(r)=\rho=N / V$ and $g\left(r_{1}, r_{2}\right)=g\left(\left|r_{1}-r_{2}\right|\right)=g(r)$ where $\rho$ is the bulk density and $g(r)$ is the radial distribution function (RDF). Then, the pressure is given by

$$
\frac{P V}{N k_{B} T}=1-\frac{\beta \rho}{6} \int d^{3} r r \frac{d u(r)}{d r} g(r),
$$

where $\left.\beta \stackrel{\text { def }}{=} 1 / k_{B} T[12]-15\right]$.

\subsection{Hard-sphere approximation}

To account for the short-distance repulsion we consider a family of trials composed by distributions that describe a gas of hard spheres of diameter $r_{d}$. For each $r_{d}$ the Hamiltonian is

$$
H_{h s}\left(q_{N} \mid r_{d}\right)=\sum_{i=1}^{N} \frac{p_{i}^{2}}{2 m}+U_{h s}
$$

with

$$
U_{h s}=\sum_{i>j}^{N} u_{h s}\left(r_{i j} \mid r_{d}\right),
$$

where

$$
u_{h s}\left(r \mid r_{d}\right)=\left\{\begin{array}{ccc}
0 & \text { for } \quad r \geq r_{d} \\
\infty & \text { for } \quad r<r_{d}
\end{array}\right.
$$


and the corresponding probability distribution is

$$
P_{h s}\left(q_{N} \mid r_{d}\right)=\frac{1}{Z_{h s}} e^{-\beta H_{h s}\left(q_{N} \mid r_{d}\right)} .
$$

The partition function and the free energy $F_{h s}\left(T, V, N \mid r_{d}\right)$ are $Z_{h s}=\int d q_{N} e^{-\beta H_{h s}\left(q_{N} \mid r_{d}\right)} \stackrel{\text { def }}{=}$ $e^{-\beta F_{h s}\left(T, V, N \mid r_{d}\right)}$. Two objections that can be raised for choosing $P_{h s}\left(q_{N} \mid r_{d}\right)$ as trials are, first, that they do not take the long-range interactions into account; and second, that the actual short range potential is not that of hard spheres. These are points to which we will return later. A third objection, and this is considerably more serious, is that the exact hard-sphere RDF is not known. However, it can be calculated within the approximation of Percus and Yevick (PY) for which there exists an exact analytical solution ([27, [28, [29]) which is reasonably simple and in good agreement with numerical simulations over an extended range of temperatures and densities, except perhaps at high densities. There are several successful proposals [30] to improve upon the PY RDF but they also represent an additional level of complication. The simpler PY RDF is sufficiently accurate for our current objective - to illustrate the application and study the broad features of the ME approach.

The PY RDF can be written in terms of the Laplace transform of $r g_{h s}\left(r \mid r_{d}\right)$ [29],

$$
G(s)=\int_{0}^{\infty} d y y g_{h s}\left(y r_{d} \mid r_{d}\right) e^{-s y}=\frac{s L(s)}{12 \eta\left[L(s)+M(s) e^{s}\right]},
$$

where $y$ is a dimensionless variable $y=r / r_{d}$,

$$
\begin{gathered}
L(s)=12 \eta\left[\left(1+\frac{1}{2} \eta\right) s+(1+2 \eta)\right], \\
M(s)=(1-\eta)^{2} s^{3}+6 \eta(1-\eta) s^{2}+18 \eta^{2} s-12 \eta(1+2 \eta),
\end{gathered}
$$

and $\eta$ is the packing fraction,

$$
\eta \stackrel{\text { def }}{=} \frac{1}{6} \pi \rho r_{d}^{3} \quad \text { with } \quad \rho=\frac{\mathrm{N}}{\mathrm{V}} .
$$

The RDF $g_{h s}\left(r \mid r_{d}\right)$ is obtained from the inverse transform using residues [31.

The equation of state can then be computed in two alternative ways, either from the "pressure" equation or from the "compressibility" equation but, since the result above for $g_{h s}\left(r \mid r_{d}\right)$ is not exact, the two results do not agree. It has been found that better agreement with simulations and with virial coefficients is obtained taking an average of the two results with weights $1 / 3$ and $2 / 3$ respectively. The result is the Carnahan-Starling equation of state, [12-15]

$$
\left(\frac{P V}{N k_{B} T}\right)_{h s}=\frac{1+\eta+\eta^{2}-\eta^{3}}{(1-\eta)^{3}} .
$$

The free energy, derived by integrating the equation of state, is

$$
F_{h s}\left(T, V, N \mid r_{d}\right)=N k_{B} T\left[-1+\ln \rho \Lambda^{3}+\frac{4 \eta-3 \eta^{2}}{(1-\eta)^{2}}\right],
$$

where $\Lambda=\left(2 \pi \hbar^{2} / m k_{B} T\right)^{1 / 2}$, and the entropy is

$$
\mathcal{D}_{h s}=-\left(\frac{\partial F_{h s}}{\partial T}\right)_{N, V}=\frac{F_{h s}}{T}+\frac{3}{2} N k_{B} .
$$

It must be remembered that these expressions are not exact. They are reasonable approximations for all densities up to almost crystalline densities (about $\eta \approx 0.5$ ). However, they fail to predict the face-centered-cubic phase when $\eta$ is in the range from 0.5 up the close-packing value of 0.74 . 


\section{The ME formalism}

\subsection{Preferred hard-sphere PDF}

As discussed in section 2, the trial $P_{h s}\left(q_{N} \mid r_{d}\right)$ that is "closest" to the "exact" $P_{f}\left(q_{N}\right)$ is found by maximizing the relative entropy $\mathcal{D}[p \mid P]$, Eq. (3), with $p=P_{h_{s}}\left(q_{N} \mid r_{d}\right)$ given by Eq. (23) and $P=P_{f}\left(q_{N}\right)$ given by Eq. (14). According to Eq. (6), it is equivalent to minimize

$$
F_{U} \stackrel{\text { def }}{=} F_{h s}+\left\langle U-U_{h s}\right\rangle_{h s}
$$

over all diameters $r_{d}$, where $\langle\cdots\rangle_{h s}$ is computed with $P_{h s}\left(q_{N} \mid r_{d}\right)$. Thus, the variational approximation to the free energy is

$$
F(T, V, N) \approx F_{U}\left(T, V, N \mid r_{m}\right) \stackrel{\text { def }}{=} \min _{r_{d}} F_{U}\left(T, V, N \mid r_{d}\right),
$$

where $r_{m}$ is the preferred diameter.

To calculate $F_{U}$ use

$$
\left\langle U-U_{h s}\right\rangle_{h s}=\frac{1}{2} \int d^{3} r d^{3} r^{\prime} n_{h s}^{(2)}\left(r, r^{\prime}\right)\left[u\left(r-r^{\prime}\right)-u_{h s}\left(r-r^{\prime} \mid r_{d}\right)\right],
$$

where $n_{h s}^{(2)}\left(r, r^{\prime}\right)=\left\langle\hat{n}^{(2)}\left(r, r^{\prime}\right)\right\rangle_{h s}$. But $u_{h s}\left(r-r^{\prime} \mid r_{d}\right)=0$ for $\left|r-r^{\prime}\right| \geq r_{d}$ while $n_{h s}^{(2)}\left(r, r^{\prime}\right)=0$ for $\left|r-r^{\prime}\right| \leq r_{d}$, therefore

$$
F_{U}=F_{h s}+\langle U\rangle_{h s}
$$

with

$$
\langle U\rangle_{h s}=\frac{1}{2} N \rho \int d^{3} r u(r) g_{h s}\left(r \mid r_{d}\right),
$$

where we have assumed that the fluid is isotropic and homogeneous, $n_{h s}^{(2)}\left(r, r^{\prime}\right)=n_{h s}^{(2)}\left(\left|r-r^{\prime}\right|\right)$, and introduced the hard-sphere RDF

$$
g_{h s}\left(r \mid r_{d}\right) \stackrel{\text { def }}{=} \frac{n_{h s}^{(2)}(r)}{\rho^{2}} .
$$

Note that the approximation does not consist of merely replacing the exact free energy $F$ by a hard-sphere free energy $F_{h s}$ which neglects the effects of long range attraction; $F$ is approximated by $F_{U}\left(r_{m}\right)$ which includes attraction effects through the $\langle U\rangle_{h s}$ term in Eq. (33). This addresses the first of the two objections mentioned earlier: the real fluid with interactions given by $u$ is not being replaced by a hard-sphere fluid. The internal energy is approximated by $\langle H\rangle_{h s}=\frac{3}{2} N k_{B} T+\langle U\rangle_{h s}$ and not by $\left\langle H_{h s}\right\rangle_{h s}=\frac{3}{2} N k_{B} T$.

To calculate $\langle U\rangle_{h s}$ it is convenient to write it in terms of $V(s)$, the inverse Laplace transform of $r u(r)$,

$$
y u\left(y r_{d}\right)=\int_{0}^{\infty} d s V(s) e^{-s y} .
$$

For example, for a Lennard-Jones potential,

$$
u(r)=4 \varepsilon\left[\left(\frac{\sigma}{r}\right)^{12}-\left(\frac{\sigma}{r}\right)^{6}\right],
$$

we have

$$
V(s)=4 \varepsilon\left[\left(\frac{\sigma}{r_{d}}\right)^{12} \frac{s^{10}}{10 !}-\left(\frac{\sigma}{r_{d}}\right)^{6} \frac{s^{4}}{4 !}\right] .
$$


Then, using equations Eq. (33) and (24) gives

$$
\langle U\rangle_{h s}=12 N \eta \int_{0}^{\infty} d s V(s) G(s) .
$$

Finally, it remains to minimize $F_{U}$ in Eq. (33) to determine the preferred diameter $r_{m}$. This is done numerically in an explicit example for argon in section 5 .

\subsection{Marginalization for optimal hard-sphere PDF}

The ME method as pursued in the last section has led us to determine a preferred hard-sphere diameter. It fails to take the softness of the repulsive core into account. As discussed in section 2.2 , our best assessment of the distribution of $q_{N}$ is given by the marginal over $r_{d}$,

$$
\bar{P}_{h s}\left(q_{N}\right) \stackrel{\text { def }}{=} \int d r_{d} P_{J}\left(q_{N}, r_{d}\right)=\int d r_{d} P_{d}\left(r_{d}\right) P_{h s}\left(q_{N} \mid r_{d}\right) .
$$

The corresponding best approximation to the RDF is obtained using Eq. (15), (18), and (35)

$$
\bar{g}_{h s}(r)=\int d q_{N} \bar{P}_{h s}\left(q_{N}\right) \hat{n}^{2}(r) / \rho^{2}=\int d r_{d} P_{d}\left(r_{d}\right) g_{h s}\left(r \mid r_{d}\right) .
$$

By averaging over all hard-sphere diameters we are effectively describing a soft-core potential. Since $\bar{g}_{h s}(r)$ takes into account soft-core effects while $g_{h s}\left(r \mid r_{m}\right)$ does not, we expect that it will lead to improved estimates for all other thermodynamic quantities.

However, we should emphasize that the distribution over the hard-sphere diameters $P_{d}\left(r_{d}\right)$ is not being introduced in an ad hoc way in order to "fix" the variational method. The introduction of $P_{d}\left(r_{d}\right)$ is mandated by the ME method (section 2.2). The distribution of diameters is given by Eq. (10)

$$
P_{d}\left(r_{d}\right) d r_{d}=\frac{e^{\mathcal{D}\left[P_{h s} \mid P\right]}}{\zeta} \gamma^{1 / 2}\left(r_{d}\right) d r_{d}=\frac{e^{-\beta F_{U}}}{\zeta_{U}} \gamma^{1 / 2}\left(r_{d}\right) d r_{d},
$$

where $\mathcal{D}\left[P_{h s} \mid P\right]=\beta\left(F-F_{U}\right)$, the partition functions $\zeta$ and $\zeta_{U}$ are given by

$$
\zeta=e^{\beta F} \zeta_{U} \quad \text { with } \quad \zeta_{\mathrm{U}}=\int \mathrm{dr} \mathrm{r}_{\mathrm{d}} \gamma^{1 / 2}\left(\mathrm{r}_{\mathrm{d}}\right) \mathrm{e}^{-\beta \mathrm{F}_{\mathrm{U}}}
$$

and the natural distance $d \ell^{2}=\gamma\left(r_{d}\right) d r_{d}^{2}$ in the space of $r_{d}$ S is given by the Fisher-Rao metric,

$$
\gamma\left(r_{d}\right)=\int d q_{N} P_{h s}\left(q_{N} \mid r_{d}\right)\left(\frac{\partial \log P_{h s}\left(q_{N} \mid r_{d}\right)}{\partial r_{d}}\right)^{2} .
$$

A convenient way to calculate the Fisher-Rao metric is to express it as a second derivative of the entropy Eq. (3) of $p=P_{h s}\left(q_{N} \mid r_{d}^{\prime}\right)$ relative to $P=P_{h s}\left(q_{N} \mid r_{d}\right)$,

$$
\gamma\left(r_{d}\right)=-\left.\frac{\partial^{2}}{\partial r_{d}^{\prime 2}} \mathcal{D}\left[P_{h s}\left(\cdot \mid r_{d}^{\prime}\right) \mid P_{h s}\left(\cdot \mid r_{d}\right)\right]\right|_{r_{d}^{\prime}=r_{d}},
$$

where

$$
\mathcal{D}\left[P_{h s}\left(\cdot \mid r_{d}^{\prime}\right) \mid P_{h s}\left(\cdot \mid r_{d}\right)\right]=\beta\left[\left.F_{h s}\right|_{r_{d}^{\prime}} ^{r_{d}}-\left\langle\left. U_{h s}\right|_{r_{d}^{\prime}} ^{r_{d}}\right\rangle_{r_{d}^{\prime}}\right],
$$

and $\langle\cdots\rangle_{r_{d}^{\prime}}$ is the average over $P_{h s}\left(q_{N} \mid r_{d}^{\prime}\right)$. As we argued above Eq. (33) the expectation of the potential energy $\left\langle U_{h s}\left(r_{d}^{\prime}\right)\right\rangle_{r_{d}^{\prime}}$ vanishes because the product $u_{h s}\left(r \mid r_{d}^{\prime}\right) g_{h s}\left(r \mid r_{d}^{\prime}\right)$ vanishes for both $r<r_{d}^{\prime}$ and $r>r_{d}^{\prime}$. Similarly, $\left\langle U_{h s}\left(r_{d}\right)\right\rangle_{r_{d}^{\prime}}=0$ when $r_{d}^{\prime}>r_{d}$. However, when $r_{d}^{\prime}<r_{d}$ the expectation 
$\left\langle U_{h s}\left(r_{d}\right)\right\rangle_{r_{d}^{\prime}}$ diverges. The divergence is a consequence of the unphysical nature of the hard-sphere model. For more realistic continuous potentials the distance between $r_{d}^{\prime}=r_{d}+d r_{d}$ and $r_{d}$ is the same as the distance between $r_{d}^{\prime}=r_{d}-d r_{d}$ and $r_{d}$. We can then always choose $r_{d}^{\prime} \geq r_{d}$ and define $\gamma\left(r_{d}\right)$ in Eq. (45) as the limit $r_{d}^{\prime}=r_{d}+0^{+}$. Then, using Eq. (29) for $F_{h s}$, we have

$$
\gamma\left(r_{d}\right)=\left.\beta \frac{\partial^{2} F_{h s}\left(r_{d}^{\prime}\right)}{\partial r_{d}^{\prime 2}}\right|_{r_{d}^{\prime}=r_{d}+0^{+}}=N \pi \rho r_{d} \frac{4+9 \eta-4 \eta^{2}}{(1-\eta)^{4}}
$$

To summarize, the distribution of diameters $P_{d}\left(r_{d}\right)$ is given by Eq. (42) with $F_{U}$ given by Equations (33), (29), (39) and $\gamma$ given by Eq. (47). Our best approximation to the "exact" $P\left(q_{N}\right)$ is the $\bar{P}_{h s}\left(q_{N}\right)$ given in Eq. (40). However, there is a problem. Since the free energy $F_{U}$ is an extensive quantity, $F_{U} \propto N$, for large $N$ the distribution $P_{d}\left(r_{d}\right) \sim \exp -\beta F_{U}$ is very sharply peaked at the preferred diameter $r_{m}$. When choosing a single preferred diameter for a macroscopic fluid sample we find that ME confers overwhelming probability to the preferred value. This is not surprising. The same thing happens when we calculate the global temperature or density of a macroscopic sample and yet local fluctuations can be important. The question then, is whether local fluctuations are relevant to the particular quantities we want to calculate. We argue that they are.

From the very definition of $g(r)$ as the probability that given an atom at a certain place another atom will be found at a distance $r$, it is clear that $g(r)$ refers to purely local behavior and should be influenced by local fluctuations. To the extent that the preferred diameter $r_{m}$ depends on temperature and density we expect that local temperature and/or density fluctuations would also induce local diameter fluctuations.

For the purpose of calculating $g(r)$ the system is effectively reduced to the small number of atoms $N_{\text {eff }}$ in the local vicinity of the reference atom at the origin. In order to develop a systematic, fully ME method for the determination of the effective number of particles $N_{\text {eff }}$ that are locally relevant, one needs to use trial probability distributions that allow inhomogeneities in the hard sphere diameters. Yet determination of such trial probability distributions requires further investigations. Since we are interested in demonstrating ME formalism for optimizing approximations in this work only, we consider a rather simple approach to estimate $N_{\text {eff }}$. Based on our ME approach to a mean field approximation for fluids [10, we have shown the RDF is given by

$$
g_{M F}(r)=e^{-\beta u(r)-\beta \rho \int d^{3} r^{\prime} u\left(r^{\prime}\right)\left(g_{M F}\left(r-r^{\prime}\right)-1\right)} .
$$

For a sufficiently dilute gas, the results of the mean field approximation are comparable with experimental results. The $g_{M F}(r)$ is approximately close to the true, exact $\operatorname{RDF} g(R)$ for $r<\sigma$, the Lennard-Jones parameter. A fluid of hard spheres gives $g\left(r \mid r_{d}\right)=0$ for $r<r_{d}$ and cannot reproduce the behavior of Eq. (48). However, once we recognize that we can use a statistical mixture, Eq. (40), we can tune the size $N_{\text {eff }}$ of the cell and thereby change the width of $P_{d}\left(r_{d}\right)$ so that the RDF $\bar{g}_{h s}(r)$ of Eq. (41) reproduces the known short-distance behavior of Eq. (48).

\section{Numerical demonstrations: Lennard-Jones "argon"}

One of the difficulties in testing theories about fluids against experimental data is that it is not easy to see whether discrepancies are to be blamed to a faulty approximation or to a wrong intermolecular potential. This is why theories are normally tested against molecular dynamics numerical simulations where there is control over the intermolecular potential. In this section we compare ME results against simulation results [18 for a fluid of monoatomic molecules interacting through a LennardJones potential, Eq. (37). The parameters $\varepsilon$ and $\sigma$ (the depth of the well, $\left.u\right|_{\min }=-\varepsilon$, and the radius of the repulsive core, $u(\sigma)=0$, respectively) are chosen to model argon: $\varepsilon=1.03 \times 10^{-2} \mathrm{eV}$ and $\sigma=3.405 \AA$. 


\subsection{Preliminary examinations}

The free energy $F_{U}$. Figure 1(A) shows the free energy $F_{U} / N k_{B} T$ as a function of hard-sphere diameter $r_{d}$ for argon at a fixed density of $\rho \sigma^{3}=0.65$ for different temperatures. Figure 1.(B) shows $F_{U} / N k_{B} T$ as a function of $r_{d}$ for several densities at fixed $T=107.82 \mathrm{~K}$. Since the critical point for argon is at $T_{c}=150.69 \mathrm{~K}$ and $\rho_{c} \sigma^{3}=0.33$ all these curves, except that at $300 \mathrm{~K}$, lie well within the liquid phase. The increase of $F_{U} / N k_{B} T$ for high values of $r_{d}$ is due to short range repulsion between the hard spheres described by $F_{h s} / N k_{B} T$. The increase for low $r_{d}$ is due to the Lennard-Jones short-range repulsion as described by $\langle U\rangle_{h s} / N k_{B} T$.

The preferred $r_{d}$ is that which minimizes $F_{U}$ and depends both on temperature and density. The preferred diameter decreases as the temperature increases because atoms with higher energy can penetrate deeper into the repulsive core. The dependence with density is less pronounced.

The distribution of diameters $P_{d}\left(r_{d}\right)$. In section 4.2 we argued that the effective number of molecules that is relevant to the local structure of the fluid is not the total number of molecules in the system $N$, but a smaller number, $N_{\text {eff }}$. In Fig 2, (A) we plot the distribution of diameters $P_{d}\left(r_{d}\right)$ for different temperatures, for a fixed fluid density of $\rho \sigma^{3}=0.65$, and for an arbitrarily chosen $N_{\text {eff }}=13500$. As expected the distribution shifts to higher diameters as the temperature decreases. Notice also that the distribution becomes narrower at lower temperatures in agreement with the fact that a hard-sphere approximation is better at low $T[12$.

Figure 2.(B) shows that increasing $N_{\text {eff }}$ (with fixed density $\rho$ ) decreases the width of $P_{d}\left(r_{d}\right)$ (solid lines) and induces a slight shift of the whole distribution. This is due to the dependence $\sim\left(N_{\mathrm{eff}} r_{d}\right)^{1 / 2}$ of the Fisher-Rao measure $\gamma^{1 / 2}\left(r_{d}\right)$ in 47. Figure 2(B) also explores the influence of $\gamma^{1 / 2}\left(r_{d}\right)$ by comparing the actual distributions $P_{d}\left(r_{d}\right)$ (solid lines) with the distributions $e^{-\beta F_{U}\left(r_{d}\right)}$ (dotted lines) which are obtained by setting $\gamma^{1 / 2}=1$ in Eq. (42). The effect of $\gamma^{1 / 2}$ is to shift the distribution slightly to higher $r_{d}$.

\subsection{Two properties of argon}

The radial distribution function. We are finally ready to calculate the radial distribution $g(r)$ for argon. We start by estimating the number of molecules $N_{\text {eff }}$ that are locally relevant; as explained earlier we choose $N_{\text {eff }}$ so that our best approximation $\bar{g}_{h s}(r)$, Eq. (41), reproduces the known shortdistance behavior $g_{M F}(r)$, Eq. (48), for $r \ll \sigma$. We have found that the estimates for $N_{\text {eff need not }}$ be very accurate but that they must be obtained for each value of the temperature and density. In Fig 3 we show an example of the short-distance behavior of $\bar{g}_{h s}$ for three values of $N_{\text {eff }}$ at $T=107.82$ $\mathrm{K}$ and $\rho \sigma^{3}=0.65$; using a Chi-square fit in the range from $r=2.9$ to $3.1 \AA$ the selected best value of $N_{\text {eff }}$ is around 38000 .

In figures 4(A)-(D) we compare three different ways to calculate the RDF. The solid line is Verlet's molecular dynamics simulation [18]; it plays the role of experimental data against which we compare our theory. The dotted line is $g_{h s}\left(r \mid r_{m}\right)$ for the hard-sphere fluid with preferred diameter $r_{m}$. This curve, calculated from inverse of Eq. (24), is also the result of the variational method and coincides with the ME result for a macroscopically large $N_{\text {eff }}=N$. The dashed line is the averaged $\bar{g}_{h s}(r)$ of the extended ME analysis. Figures. 4.(A)-(C) were plotted at three different temperatures $T=107.82,124.11$ and $189.76 \mathrm{~K}$ at the density $\rho \sigma^{3}=0.65$. Figure 4 (D) we changed the density and the temperature to $\rho \sigma^{3}=0.5$ and $T=162.93 \mathrm{~K}$. The agreement between the ME curve and Verlet's data is good. The vast improvement over the simpler variational method calculation is clear.

One might be tempted to dismiss this achievement as due to the adjustment of the parameter $N_{\text {eff }}$ but this is not quite correct: $N_{\text {eff }}$ has not been adjusted, it has been calculated by fitting $\bar{g}_{h s}(r)$ to optimal mean field $\mathrm{RDF} g_{M F}(r)$ for $r<\sigma$. Indeed, despite the fact that the hard-sphere trial solutions that we employ are mere approximations, the functional form of the whole curve $\bar{g}_{h s}(r)$ in Eq. (41) is reproduced quite well.

However, one may note that the agreement between the ME prediction and Verlet's data becomes worse when the fluid density is decreased or the temperature is increased. The reason has been spelled 
out by in the studies of WCA [13] They demonstrate that both the repulsive and attractive forces contribute to the fluid structure when fluid is at low and moderate densities $\left(0.4 \lesssim \rho \sigma^{3} \lesssim 0.65\right)$. However, when the fluid density is high enough $\left(\rho \sigma^{3} \gtrsim 0.65\right)$, the repulsive force becomes dominant. Because the hard-sphere approximation does not include the attractive force, the hard-sphere RDF does not take the attractive force into account, and this error propagates into our ME prediction. The same discrepancy is also revealed in the WCA theory for low density [13].

The equation of state. Finally we use the RDF to calculate the equation of state from the pressure equation, Eq. (19). In Fig 5 we compare the equation of state derived from the $g(r)$ obtained from Verlet's simulation with calculations using the EME and variational methods and the perturbative theories of Barker and Henderson [12] and of Weeks, Chandler and Anderson [13, at $T=161.73$ $K$. The EME results constitute a clear improvement over the plain variational calculation. For low densities all four methods agree with each other but differ from the simulation. A better agreement in this region would probably require a better treatment of two-particle correlations at long distances. At intermediate densities the best agreement is provided by the EME and $\mathrm{BH}$ results, while the WCA theory seems to be the best at high densities. Also shown in Fig 5 (A) are experimental data on argon [32. The discrepancy between the experimental curve and the Verlet simulation is very likely due to the actual potential not being precisely of the Lennard-Jones type.

In Fig 5. (B) we plot the EME equation of state for three different isotherms $(T=137.77,161.73$ and $328.25 \mathrm{~K}$ ). To compare to the simulation of Hansen and Verlet [19] we plot $\beta P$ (rather than $\beta P / \rho)$ as a function of density $\rho \sigma^{3}$ because this kind of plot exhibits the characteristic van der Waals loop that signals the liquid-gas transition as the temperature drops. A more exhaustive exploration lies, however, outside the scope of this paper.

\section{Conclusion}

The goal of this paper has been to use the EME method to generate approximations and show that this provides a generalization of the Bogoliubov variational principle. This addresses a range of applications that lie beyond the scope of the traditional MaxEnt. To test the method we considered simple classical fluids.

When faced with the difficulty of dealing a system described by an intractable Hamiltonian, the traditional approach has been to consider a similar albeit idealized system described by a simpler more tractable Hamiltonian. The approach we have followed here departs from this tradition: our goal is not to identify an approximately similar Hamiltonian but rather to identify an approximately similar probability distribution. The end result of the EME approach is a probability distribution which is a sum or an integral over distributions corresponding to different hard-sphere diameters. While each term in the sum is of a form that can be associated to a real hard-sphere gas, the sum itself is not of the form exp $-\beta H$, and cannot be interpreted as describing any physical system.

As far as the application to simple fluids is concerned the results achieved in this paper represent progress but further improvements are possible by using better approximations to the hard-sphere fluid and by choosing a broader family of trial distributions. An important improvement would be to use trial probability distributions that allow inhomogeneities in the hard sphere diameters. This would lead to a systematic, fully EME method for the determination of the effective number of particles $N_{\text {eff }}$ that are locally relevant.

Many perturbative approaches to fluids had been proposed, and a gradual process of selection over many years of research led to the optimized theories of BH and WCA. The variational approach was definitely less satisfactory than these "best" perturbation theories. With our work, however, the situation has changed: the EME-improved variational approach offers predictions that already are competitive with the best perturbative theories. And, of course, the potential for further improvements of the EME approach remains, at this early date, far from being exhausted. 


\section{Acknowledgements}

The authors acknowledge R. Scheicher and C.-W. Hong for their valuable assistance and advice with the numerical calculations.

\section{References}

[1] N. N. Čencov: Statistical Decision Rules and Optimal Inference, Transl. Math. Monographs, vol. 53, Am. Math. Soc. (Providence, 1981);L. L. Campbell, Proc. Am. Math. Soc. 98 (1986) 135; for applications to statistics see S. Amari, Differential-Geometrical Methods in Statistics, Springer-Verlag (New York, 1985); S. Amari and H. Nagaoka, Methods of Information Geometry, Oxford (Providence, 2000); for a brief derivation see A. Caticha, Change, time and information geometry, in: A Mohammad-Djafari (ed), Bayesian Methods and Maximum Entropy in Science and Engineering, AIP Conf. Proc. 568, 2001 (arXiv:math-ph/0008018).

[2] J. E. Shore, R. W. Johnson, IEEE Trans. Inf. Theory IT-26 (1980) 26; J. E. Shore, R. W. Johnson, IEEE Trans. Inf. Theory IT-27 (1981) 472.

[3] J. Skilling, The Axioms of Maximum Entropy, in: G. J. Erickson, C. R. Smith (Eds), MaximumEntropy and Bayesian Methods in Science and Engineering, Dordrecht, Kluwer, 1988.

[4] J. Skilling, Classic Maximum Entropy, in: J. Skilling (Ed), Maximum Entropy and Bayesian Methods, Dordrecht, Kluwer, 1989.

[5] J. Skilling, Quantified Maximum Entropy, in: P. F. Fougère (Ed), Maximum Entropy and Bayesian Methods, Dordrecht, Kluwer, 1990.

[6] I. Csiszar, Ann. Stat. 19 (1991) 2032.

[7] A. Caticha, Relative Entropy and Inductive Inference, in: G. Erickson, Y. Zhai (eds), Bayesian Inference and Maximum Entropy Methods in Science and Engineering, AIP Conf. Proc. 707, 2004 (arXiv:physics/0311093); A. Caticha and A. Giffin, Updating Probabilities, in: K.H. Knuth, A. Caticha, A. Giffin, C. C. Rodriguez, J. L. J. Center (eds.), Bayesian Inference and Maximum Entropy Methods in Science and Engineering, AIP Conf. Proc. 954, 2007 arXiv:physics/0608185v1 ) .

[8] A. Caticha, Lectures on Probability, Entropy, and Statistical Physics, in MaxEn08, São Paulo, 2008 (arXiv.org/abs/0808.0012); an application to data analysis is given in A. Caticha and R. Preuss, Phys. Rev. E 70 (2004) 0461271.

[9] E. T. Jaynes, Phys. Rev. 106 (1957) 620; E. T. Jaynes Phys. Rev. 108 (1957) 171; E. T. Jaynes in: R D Rosenkrantz (ed), E. T. Jaynes: Papers on Probability, Statistics and Statistical Physics, Dordrecht (Reidel, 1983); E. T. Jaynes: Probability Theory: The Logic of Science, Cambridge University Press (Cambridge, 2003).

[10] C.-Y. Tseng, A. Caticha, Maximum Entropy approach to a Mean Field Theory for Fluids. in: C. J. Williams (Ed), Bayesian Inference and Maximum Entropy Methods in Science and Engineering, AIP Conf. Proc. 659, 2003 (arXiv:cond-mat/0212198).

[11] C.-Y. Tseng, A. Caticha, Maximum Entropy Approach to the Theory of Simple Fluids, in: G. Erickson and Y. Zhai (Eds), Bayesian Inference and Maximum Entropy Methods in Science and Engineering, AIP Conf. Proc. 707, 2004 (arXiv:cond-mat/0310746).

[12] J. A. Barker, D. Henderson, Rev. Mod. Phys. 48 (1976) 587. 
[13] J. D. Weeks, D. Chandler, H. C. Andersen, J. Chem. Phys. 54 (1971) 5237; J. D. Weeks, D. Chandler, H. C. Andersen, Science 220 (1983) 787.

[14] J. P. Hansen, I. R. McDonald: Theory of Simple Liquids, 2nd edition, Acad. Press (London, 1986).

[15] V. I. Kalikmanov: Statistical Physics of Fluids, Springer (New York, 2002).

[16] G. A. Mansoori, F. B. Canfield, J. Chem. Phys. 51 (1969) 4958.

[17] Caticha A, Maximum entropy, fluctuations and priors, in: A Mohammad-Djafari (ed), Bayesian Inference and Maximum Entropy Methods in Science and Engineering, AIP Conf. Proc. 568, 2001 (arXiv:math-ph/0008017).

[18] L. Verlet, Phys. Rev. 165 (1968) 201.

[19] J. P. Hansen and L. Verlet, Phys. Rev. 184 (1961) 151.

[20] P. Germain and S. Amokrane, Phys. Rev. E 65 (2002) 0311091.

[21] M. López de Haro, M. Robles, Physica A 372 (2006) 307.

[22] C. Valeriani, Z.-J. Wang and D. Frenkel, Mol. Sim. 33 (2007) 1023.

[23] T. Urbic, V. lachy, Yu. V. Kalyuzhnji and K. A. Dill, J. Chem. Phys. 127 (2007) 174511.

[24] A. B. Adib, Phys. Rev. E 75(2007) 061204.

[25] F. F. Betancourt-Cárdenas, L. A. Galicia-Luna and S. I. Sandler, Fluid Phase Equilibria 264 (2008) 174.

[26] H. B. Callen: Thermodynamics and an Introduction to Thermostatistics, Wiley (New York, 1985).

[27] J. K. Percus and G. J. Yevick, Phys. Rev. 110 (1958) 1.

[28] J. K. Percus, Phys. Rev. Lett. 8 (1962) 462.

[29] M. S. Wertheim, Phys. Rev. Lett. 10 (1963) 321; M. S. Wertheim, J. Math. Phys. 5 (1964) 643;

E. Thiele, J.Chem. Phys. 39 (1963) 474.

[30] S. Bravo Yuste and A. Santos, Phys. Rev. A 43 (1991) 5418; S. Bravo Yuste, M. López and A. Santos, Phys. Rev. E 53 (1996) 4820-; Y. Tang and B. C.-Y. Lu, J. Chem. Phys. 103 (1995) 7463.

[31] G. J. Throop and R. J. Bearman, J. Chem. Phys. 42 (1965) 2408.

[32] J. M. H. Levelt, Physica 26 (1960) 361. 
(A)

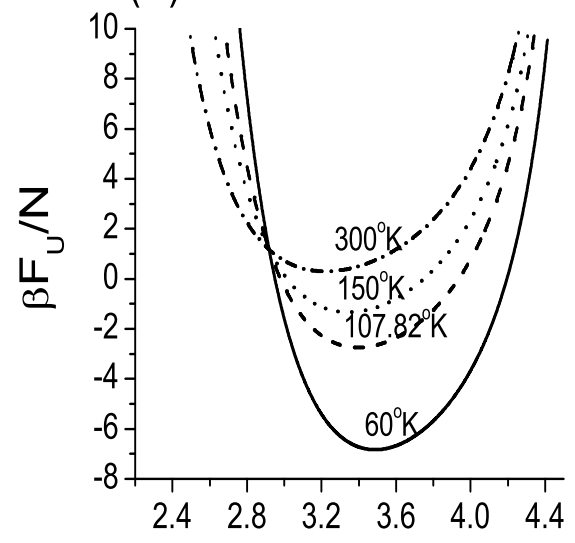

(B)

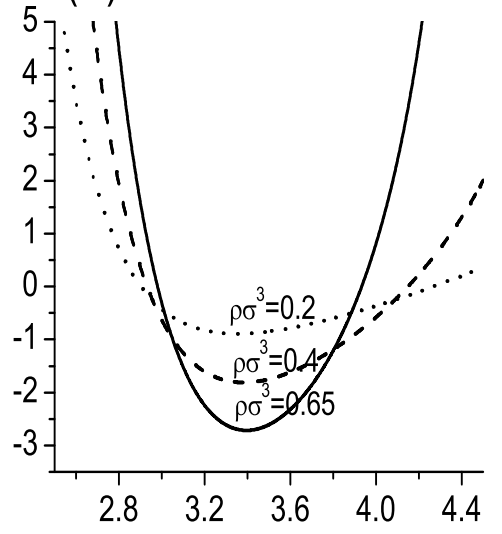

$r_{d}(A)$

Figure 1: (A): The free energy $F_{U}$ as a function of hard-sphere diameter $r_{d}$ for argon at a density of $\rho \sigma^{3}=0.65$ for different temperatures. The best $r_{d}$ is that which minimizes $F_{U}$. (B): $F_{U}$ as a function of $r_{d}$ for argon at $T=107.82 \mathrm{~K}$ for different densities. 

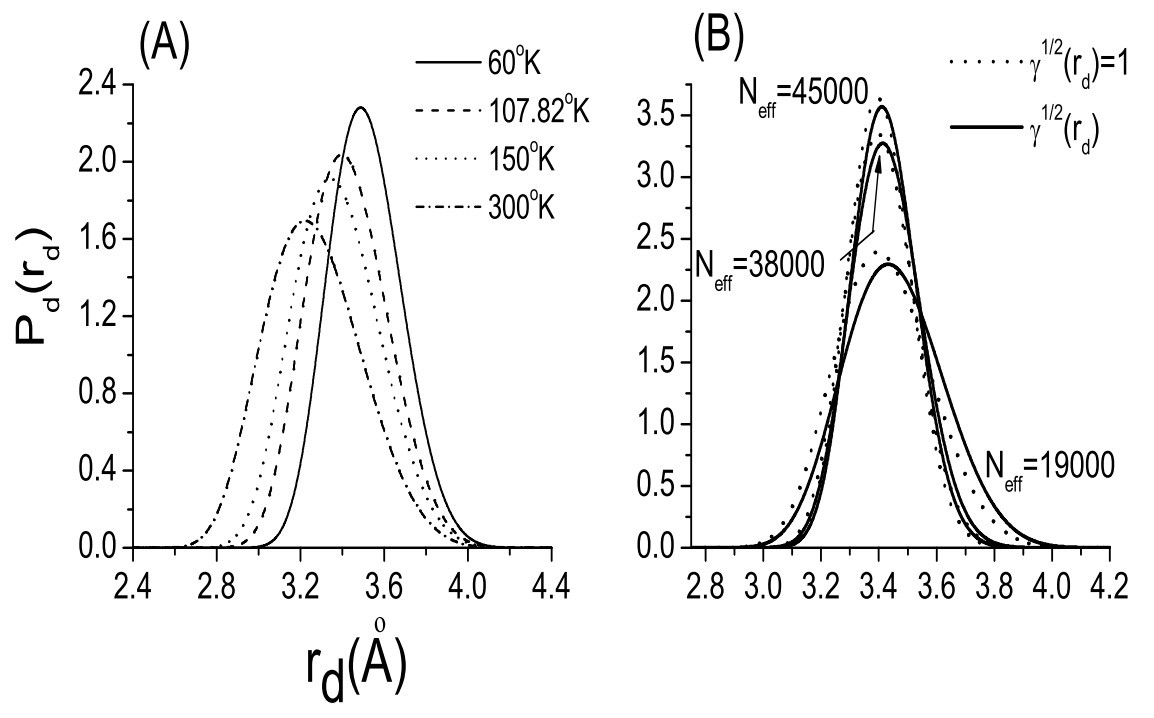

Figure 2: (A): The distribution of hard-sphere diameters $r_{d}$ for argon for several temperatures at density $\rho \sigma^{3}=0.65$ for $N_{\text {eff }}=13500$. (B): $P_{d}\left(r_{d}\right)$ for various $N_{\text {eff }}$ at $T=107.82 \mathrm{~K}$ and $\rho \sigma^{3}=0.65$. By setting $\gamma^{1 / 2}=1$ (dotted lines) we see that the effect of the $\gamma^{1 / 2}$ factor is to cause a slight shift of the distribution. 


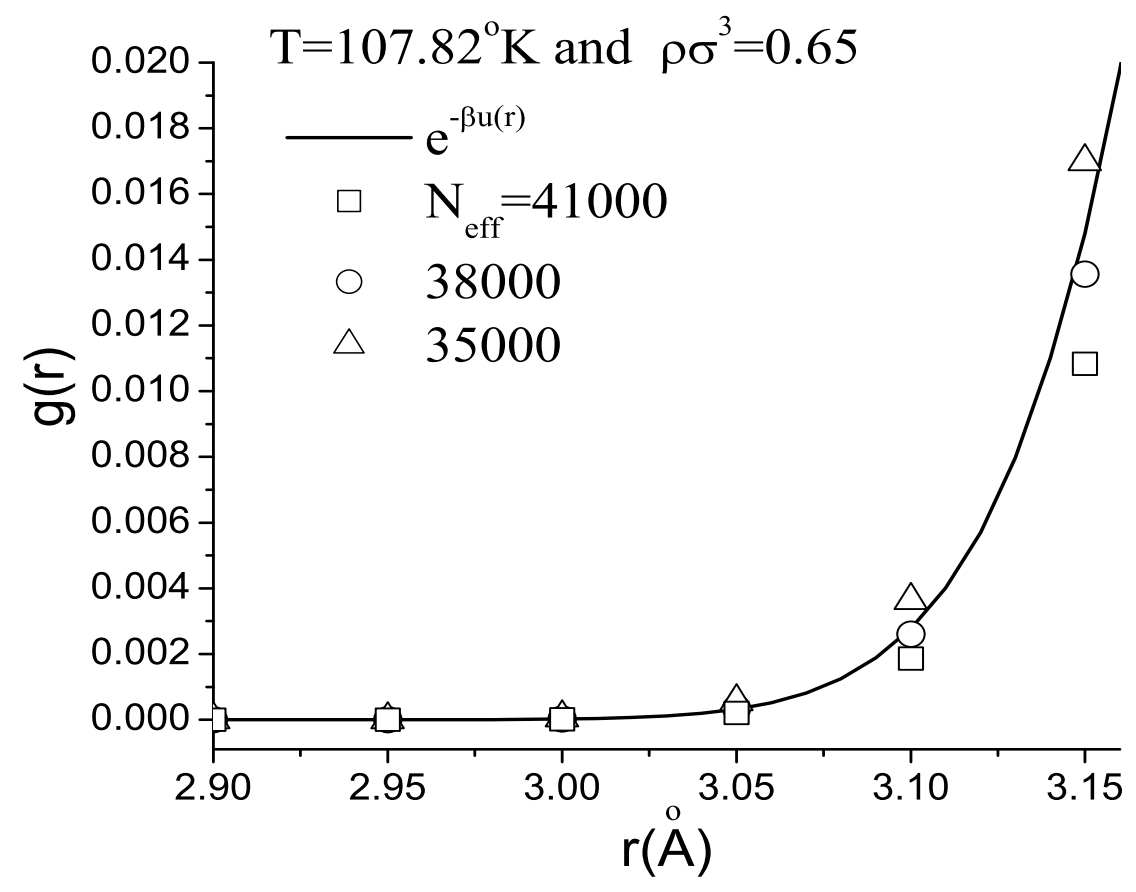

Figure 3: Estimating $N_{\text {eff }}$ by requiring that $\bar{g}_{h s}(r)$ have the correct short-distance behavior $e^{-\beta u(r)}$. 
(A)

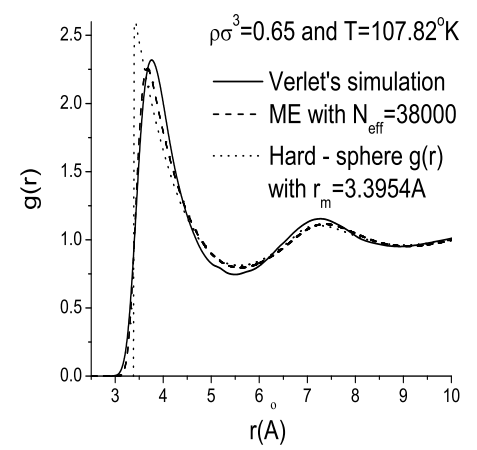

(C)

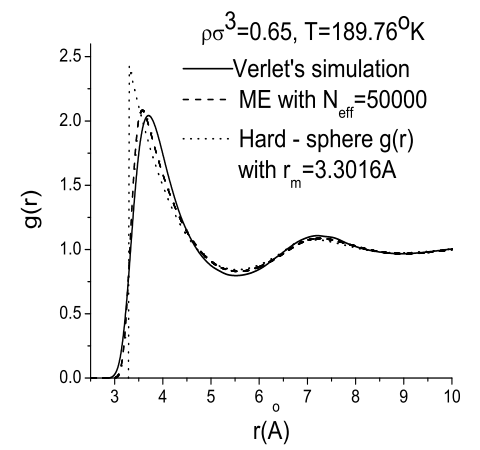

(B)

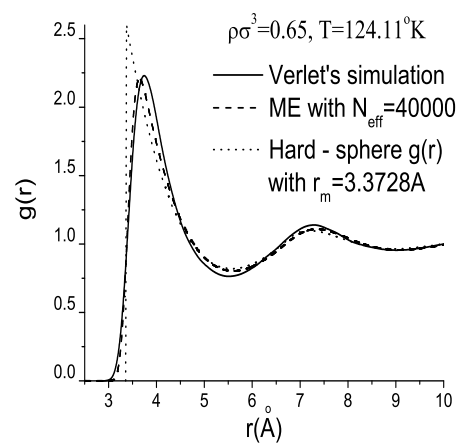

(D)

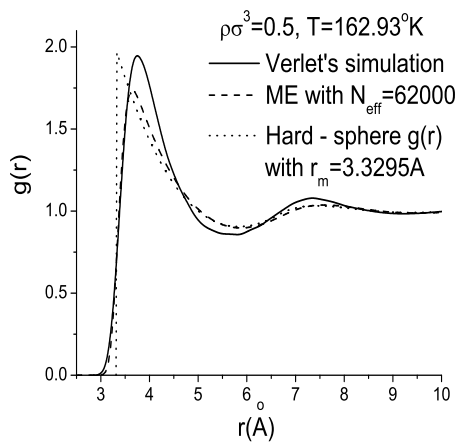

Figure 4: The radial distribution function for (a) the hard-sphere fluid with optimal diameter $r_{m}$; (b) Verlet's molecular dynamics simulation; and (c) the improved EME analysis, for argon at (A): density $\rho \sigma^{3}=0.65$, temperature $T=107.82 \mathrm{~K}$, and effective particle number $N_{\text {eff }}=38000$. (B): $\rho \sigma^{3}=0.65, T=124.11 \mathrm{~K}$, and $N_{\text {eff }}=40000 .(\mathrm{C}): \rho \sigma^{3}=0.65, T=189.76 \mathrm{~K}$, and $N_{\text {eff }}=50000$. (D): $\rho \sigma^{3}=0.5, T=162.93 \mathrm{~K}$, and $N_{\text {eff }}=62000$. 


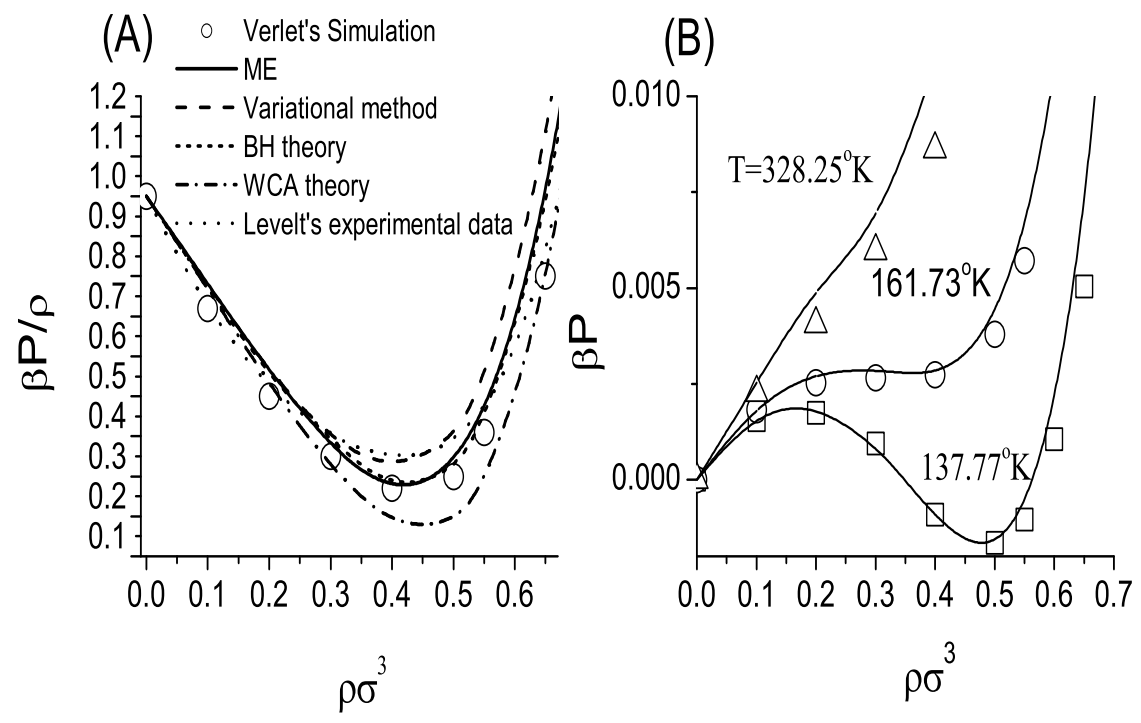

Figure 5: (A): The argon equation of state calculated using the EME method, the variational method and the perturbative theories of BH and WCA are compared to the Verlet simulation at $T=161.73$ K. Also shown are Levelt's experimental results. (B): $\beta P$ versus the reduced density $\rho \sigma^{3}$ calculated using the EME method (solid line) and compared to the Hansen-Verlet simulation for three different isotherms. The graph shows the appearance of the liquid-gas van der Waals loop as the temperature drops. 